\title{
Machine Learning for the Prediction of Springback in High Tensile Strength Steels after V-Bending Process Using Tree-Based Learning
}

\section{Saad Ur Rehman Baig ( $\sim$ saadbaig200@gmail.com )}

NED University of Engineering \& Technology https://orcid.org/0000-0001-5327-5540

Muhammad Wasif

NED University of Engineering and Technology

Anis Fatima

NED University of Engineering and Technology

Mirza Muhammad Anas Baig

Karachi, Pakistan

Syed Amir lqbal

NED University of Engineering and Technology

\section{Research Article}

Keywords: Machine learning, V-bending, springback, High tensile strength Steel, tree-based learning algorithms, Sheet metals

Posted Date: August 10th, 2021

DOl: https://doi.org/10.21203/rs.3.rs-795174/v1

License: (c) (1) This work is licensed under a Creative Commons Attribution 4.0 International License. Read Full License 


\title{
Machine Learning for the Prediction of Springback in High Tensile
}

\section{Strength Steels after V-Bending Process Using Tree-Based Learning}

\author{
Saad Ur Rehman Baig ${ }^{* 1}$, Muhammad Wasif ${ }^{1}$, Anis Fatima ${ }^{1}$, Mirza Muhammad Anas Baig ${ }^{2}$, \\ Syed Amir Iqbal ${ }^{1}$ \\ ${ }^{1}$ Department of Industrial and Manufacturing Engineering (IMD), \\ NED University of Engineering and Technology, Karachi. \\ ${ }^{2}$ Karachi, Pakistan. \\ *saadbaig200@gmail.com, Ph: +92 3309223344
}

\begin{abstract}
Sheet metal bending is a typical operation and springback is an unintended consequence of this operation. Since it causes fitting issues in the assembly, which leads to quality problems, anticipating it long before the bending operation is done is essential in today's production, so that machining parameters can be adjusted accordingly. In order to predict springback with minimum errors, this paper presents the idea for the development of machine learning models using tree-based learning algorithms (A class of machine learning algorithms). Tree-based learning algorithms are employed because they are precise, consistent, and easy to understand. Experimental studies provided the data for training and testing the models. The model's input parameters were sheet Material, Thickness, Width, Initial Angle (Desired angle), and Machine used to perform the bending. Following the training and testing of different tree-based learning algorithms, the results were evaluated using MAE and MSE. It was determined that Gradient boosting algorithms (a class of tree-based learning) gave the best results. Later on further evaluation of algorithms, it was found that LightGBM produced the best results, with MAE and MSE of 0.41 and 0.25 , respectively.
\end{abstract}

\section{Keywords}

Machine learning; V-bending; springback; High tensile strength Steel; tree-based learning algorithms; Sheet metals.

\section{Introduction}

The use of sheet metal has tremendously increased due to its application in domestic and commercial appliances. By definition, sheet metal is any form of metal that has a relatively large length to thickness ratio, their thickness typically ranges from $0.4 \mathrm{~mm}$ to $6 \mathrm{~mm}$ [1]. Sheet-metals parts are formed for different kinds of applications and are now required in almost all types of equipment, whether domestic or commercial. Automobile original equipment manufacturers (OEMs) around the globe use hightensile strength steel sheets because it provides increased strength-to-weight ratio and corresponding toughness to vehicles. Due to this reason, it is primarily used in structural parts as it provides safety and improved fuel efficiency [2]. Parts made of sheet metals are manufactured by various processes like piercing, drawing, shaping, bending, etc. Among these, the most applied manufacturing process on sheet metal is bending [3].

Bending is forming operation in which the sheet metal is forced to acquire the shapes of the cavity formed between the punch and the die. The load applied is beyond its yield strength but below its ultimate tensile strength, such that a permanent deformation is made. During this process, the metal on 
the outside of the neutral plane is stretched, while the metal on the inside of the neutral plane is compressed, as shown in Fig 1.

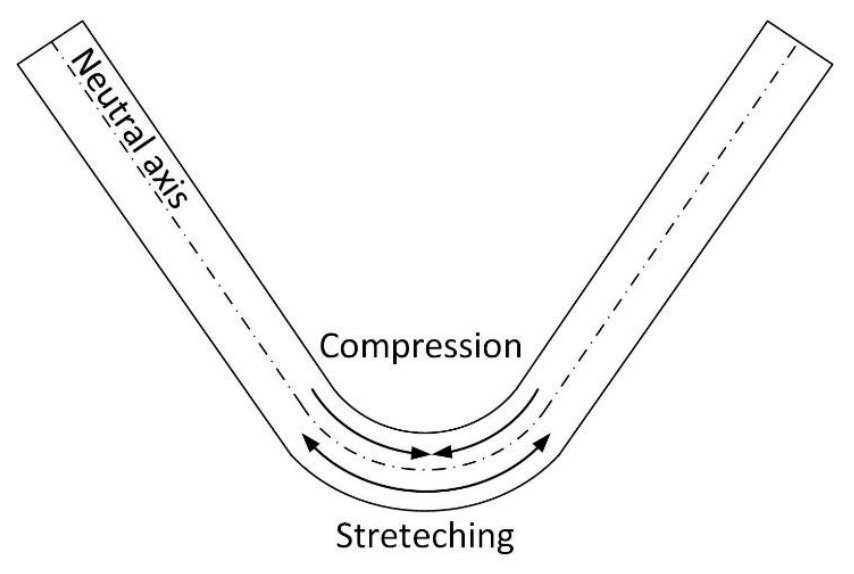

Fig 1 Compression and tensile elongation of the metal in bending

V-bending is one of the most common type of bending operation in which the sheet-metal part produced have a V-shape as shown in Fig 2(b) and a bend angle ranging from obtuse to acute including right angle.
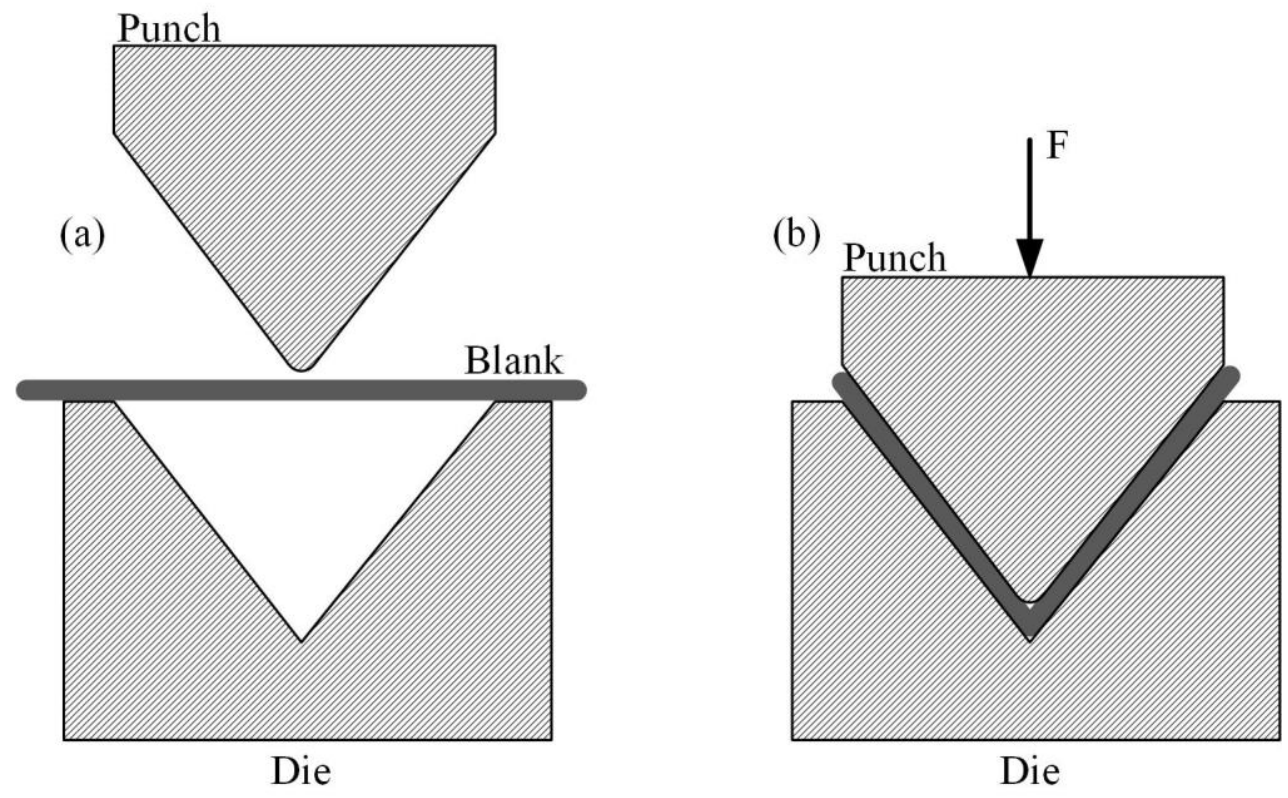

Fig 2 Illustration of V-bending (a) before and (b) after bending

As illustrated in Fig 2, the punch (male part) having a concave shape pushes the sheet metal into the die (female part) having a convex shape. It causes the metal to acquire the shape of the die. When the punch is pulled back from the sheet metal, it tends to recover due to its elastic behaviour. This elastic recovery is termed as springback, as illustrated in Fig 3. Depending on the tensile nature of the material, springback can either be positive (i.e., when sheet metal recovers outward) or negative (i.e., when the sheet metal recovers inward) 


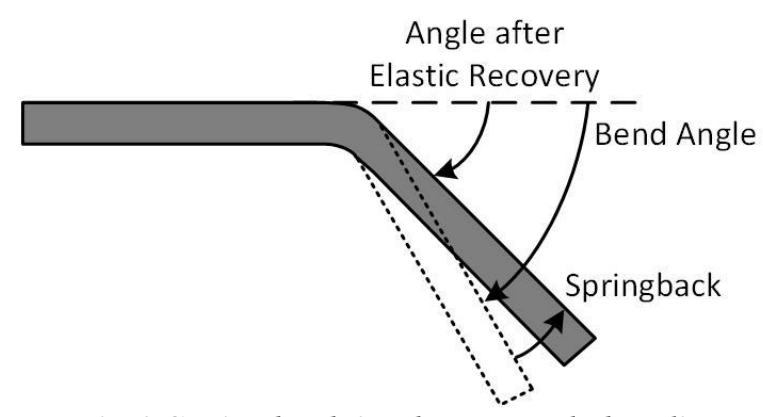

Fig 3 Springback in sheet-metals bending

With the rapid development in manufacturing and increasing demand for high-quality products, the requirement to produce parts with high precision and accuracy has become a need of the manufacturing world, since it is known that springback is an undesired outcome, so the need for minimizing this in sheet metal parts is of utmost importance, this could not be achieved by adopting traditional approaches to predicting springback, so a newer approach known as a machine learning approach is adopted.

Machine learning is a branch of Artificial intelligence in which, given input data points and output value, a computer algorithm learns rules by Analyzing the data. In other words, it gives systems the ability to learn and improve themselves without explicitly being programmed. The recent advancement in technology and the development of manufacturing 4.0 also triggered the need for machine learning. It means that the machines are producing data at an unprecedented scale, so now it is needed to have fast learning algorithms that can give accurate results in a short amount of time. This need triggered engineers worldwide to build new sets of algorithms that are fast at learning and can also give reliable answers. One such group of algorithms already exist which are known as tree-based learning algorithms. A tree-based learning algorithm is a group of machine learning algorithms that are used for supervised learning.

An extensive literature review was conducted to study the effects of springback in the V-bending process during mass production.

Cardena et al. based on data collected from experimental settings created a link between the springback of steel and aluminium alloys [4]. They concluded that as the tensile stress approaches the yield stress, the springback can be minimized. In another research, Tekiner investigated various high tensile strength sheet metals to study the influence of springback in the air bending process using workpieces of multiple thicknesses on modular dies [5]. Fei and Hodgson, in their investigation, presented that in TIRP steel, the influence of the coefficient of friction and the change in Young's modulus due to plastic deformation have vital roles in the springback [6]. Dong-Juan et al. in their paper took into consideration the effects of length of bending arm between the punch and die, neutral surface shifting, transverse stress, sheet thickness thinning on the sheet, and contact pressure and presented an analytical model based on Hill's yielding criterion and plane strain condition, to predict springback after V-bending process [7]. Ozturk et al. conducted an experimental investigation on steel sheets of DP600 at temperatures ranging from room temperature to $300^{\circ} \mathrm{c}$ to explore the behaviours of springback [8]. Inamdara et al. used variance (ANOVA) analyzing the data obtained from performing air V-bending on different materials to investigate the effects of springback due to tools geometry, workpiece geometry, and material properties [9]. Ramezani et al. used the finite element modelling (FEM) method to investigate springback in the V-bending process by modelling kinetic friction of ultra-high-tensile steels [10]. Swiatoniowski and Balon provided a model to determine the best workpiece and correct deviations in workpiece caused by springback in parts using sheet metal [11]. Slota and Jurcisin built a prediction model for springback using the FEM method to investigate the behaviour of steels in the air bending process [12]. Da Silva et al. conducted a comparative investigation to compare springback between advanced high-tensile strength material with existing high-tensile strength materials [13]. Yang et al. developed a model to predict springback in DP780 (Advanced High-Strength Steel) after the air bending process, which is very hard to foresee because of fluctuating Young's moduli during the stress application [14]. Jung et 
al. built a model by studying the anisotropic behaviour of dual-phase steel (advanced high-tensile strength steels) to predict springback in $U$ bending process. This model was validated through a series of experiments [15]. Choi et al. investigated U-bending in high tensile steel using FEM-based simulations. They noticed that loading had a non-proportional influence on the springback [16].

Based on numerical simulation approaches, Leu and Zhuang predicted the springback of high tensile strength materials by developing an analytical model. The model's characteristics focus on the sheet thickness, material strength, and punch radius [17]. Cai et al. experimental investigation presented the effects on the springback at varying temperatures on high tensile strength aluminium alloy (AA6082) during the U-bending process. It was found that during U-bending, the springback was minimized at increased temperatures [18].

Nakagawa et al. investigated the impact on springback during stamping and subsequent quenching process in ultra high strength steel sheets. By adopting the holding technique during the stamping process, he was able to lessen the springback in many ways. [19]. By incorporating the strength differential (SD) model generated by the experiments into the FEM model, Noma et al. conducted a finite element modelling (FEM) based research of springback in sheet metals. [20]. Liu et al. introduced a new springback model based on FEM and investigated the springback during the layer-by-layer milling process in stiffed panels. Those researchers also used milling experiments to validate the springback model [21]. Liu et al. presented a study on the FEM investigation of springback relating to titanium square tubes, with the results being experimentally validated. Researchers observed that increase in springback is due to the radius of bent tubes [22]. Ramadass et al. developed a FEM model to minimize the springback in Titanium alloyed Steel sheets. They also applied Taguchi orthogonal array to reduce the noise ratio and optimized the controllable parameters for the minimum springback [23]. Lin et al., in another research, proposed a FEM model to predict springback and verify it through the experiments in conduit parts of MP980 and AA6022-T4. They concluded that including yield criteria in the FEM model improves the springback prediction [24].

Inamdar et al. developed an artificial neural network to predict the springback and punch travel for the air V-bending process, which could be used to get the desired angle in a single stroke [25]. Dezelak, et al. used a machine learning approach for updating the parameters of FEM models of springback and they found the combined models had better accuracy than FEM alone [26]. Narayanasamy and Padmanabhan presented a comparative investigation to compare the prediction of springback between regression and Artificial neural network models during the air bending process of interstitial free steel sheet. It was found that ANN had higher accuracy than the regression model [27]. Kazan et al. predicted the springback in the wipe bending process by using an artificial neural networks approach to developing a model and training that model using the data obtained from FEM [28].

The literature review shows that FEM methods and Machine Learning approaches are the two techniques that are vastly applied to predict the springback in sheet metals. Since FEM is slow so it cannot be used as an on-line tool in the production line for predicting springback [29]. In machine learning, most of the earlier attempts used artificial neural networks (ANN) to predict springback, which has several limitations. Using ANN, the predictions cannot be justified easily, i.e., the explainability of the answer from the neural network is very low. Neural networks require a lot of computation power to train the model. A neural network needs a large amount of data so that the model trained is generalized, rather than overfitted or under fitted to the data.

Hence, this research article used tree-based learning algorithms which have high explainability, needs less computational power, and need less data to train the model. Data has been acquired through the experimental setup developed for the v-bending of high-tensile strength sheet metals. Sheet metals are bend to 60,90, and 120 degrees, after which final angles are measured through the digital bevel gauge. The data is cleaned and input into the machine learning algorithm, which developed the algorithm model. The model is assessed for performance and accuracy and the results are presented. The overall 
objective of the research is to predict the springback in the high-tensile strength sheet metals for the given parameters such as bend angle, width and thickness of the sheet.

This article is divided into four sections. Section 1 presents the literature review, problem statement and the objectives of the research. The second section presents the research methodology, whereas the third section addresses the results and the discussion. The final section shows the outcomes and conclusion of the research.

\section{Methodology}

A new framework for the prediction of springback based on machine learning is developed. The proposed method is designed to assist in developing predictive models from data acquired through the experimental setup. To cover the core objective of the research, the following step-wise methodology (see Fig 4) has been adopted, which is discussed in detail in the section that follows.

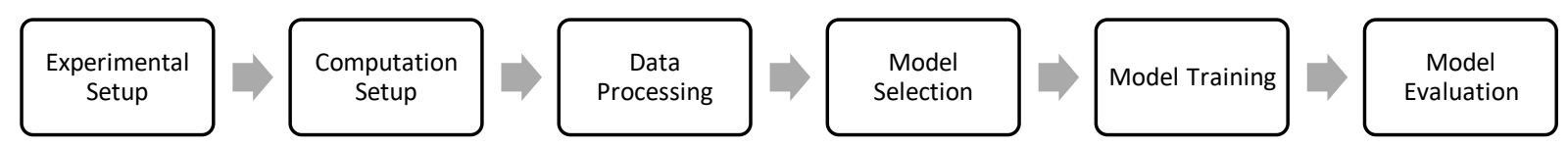

Fig 4 Methodology of the research

\subsection{Experimental Setup}

For the collection of springback data, bending experiments were performed on four types of steel sheets which are JSC440, JSC590, JSH440 and JSH590. The process and geometric parameters of the workpieces are mentioned in Table 1. A mechanical press of 60 tons and a hydraulic press of 75 tons with varying pressure of $30 \mathrm{~kg} / \mathrm{cm}^{2}$ and $180 \mathrm{~kg} / \mathrm{cm}^{2}$ are used. No-load holding time is applied while bending the sheet metals using the mechanical press, whereas 0 second and 10 -second holding times are applied while using the hydraulic press. The gap between punch and die is also varied, to include this in the prediction model. The gap between the dead-end of punch and die is set to equal the thickness of the sheet or 0.7 times the thickness while bent with the mechanical press. At the same time, it is kept equal for the hydraulic press. Sheet metals of thicknesses $1 \mathrm{~mm}, 1.2 \mathrm{~mm}, 1.4 \mathrm{~mm}$ and $1.6 \mathrm{~mm}$ are used, whereas the widths of the sheets are $20 \mathrm{~mm}$ and $50 \mathrm{~mm}$. The sheet metals are bend to 60,90 and 120 degrees.

After V-bending operations are applied, the final bend angle of the sheet metal is measured using a well-calibrated digital bevel gauge of least count up to 1 minute. The springback in the sheet metals is determined by subtracting the measured angle from the desired angle. This process was repeated for 428 samples with two replication of various materials, thickness, desired angle, load holding time, the gap between punch and die, and width. These varying parameters are given in Table 1 and constant parameters are shown in Table 2. The length of the sheet metal blank, internal bend radius and external, bend radius is set to constant. 
Table 1 Varying Parameters

\begin{tabular}{lcc}
\hline \multicolumn{1}{c}{ Parameters } & For Mechanical Press & Value \\
\hline Load Tonnage (T) & 60 tons & For Hydraulic Press \\
Load holding time (HT) & None & 75 tons with \\
Gap between punch and die (G) & Equal to blank thickness $(\mathrm{t})$ & $\begin{array}{c}\left.\mathrm{L}=30 \mathrm{~kg} / \mathrm{cm}^{2} \text { and } \mathrm{L}=180 \mathrm{~kg} / \mathrm{cm}^{2}\right) \\
0 \text { seconds, } 10 \text { seconds }\end{array}$ \\
Blank Material & Equal to 0.7t & JSC440, JSC590, JSH440, JSH590 thickness (t) \\
Thickness of blank (t) & $1.0 \mathrm{~mm}, 1.2 \mathrm{~mm}, 1.4 \mathrm{~mm}, 1.6 \mathrm{~mm}$ \\
Width of blank (w) & \multicolumn{2}{c}{$20 \mathrm{~mm}, 50 \mathrm{~mm}$} \\
Band angle $(\theta)$ & \multicolumn{2}{c}{$60^{\circ}, 90^{\circ}$ and $120^{\circ}$} \\
\hline
\end{tabular}

Table 2 Constant Parameters

\begin{tabular}{lc}
\hline \multicolumn{1}{c}{ Parameters } & Value \\
\hline Blank Length & $170 \mathrm{~mm}$ \\
Internal Bend Radius/Punch Radius & $8 \mathrm{~mm}$ \\
External Bend Radius/Die Radius & $10 \mathrm{~mm}$ \\
\hline
\end{tabular}

\subsection{Computation Setup}

For training machine learning models MacBook Pro (13-inch, 2017) having a 3.1 GHz Dual-Core Intel Core i5 processor, 8 GB 2133 MHz LPDDR3 memory, and Intel Iris Plus Graphics 6501536 MB graphics. The operating system used is Big Sur. The code for the model is written in python 3.8.5 using the IDE called jupyter notebook from Anaconda 4.9.2. The libraries used are mentioned in Table 3.

Table 3 Libraries Used in The Experiment

\begin{tabular}{lc}
\hline \multicolumn{1}{c}{ Name } & Version \\
\hline Pandas & 1.1 .3 \\
NumPy & 1.19 .2 \\
Scikit-learn & 0.23 .2 \\
Matplotlib & 3.3 .2 \\
LightGBM & 3.2 .1 \\
Flaml & 0.3 .6 \\
\hline
\end{tabular}

\subsection{Data Preprocessing}

Although the dataset collected from the experimental setup includes seven features (parameters), the first three parameters; load tonnage, load holding time, and gap between the punch and the die, are combined to form a single feature, "Machine". It is because different combinations of these factors may be combined to form a single machine setting parameters. The other parameters are; blank material, thickness, width and initial bend angle. Hence, there are five features of the dataset, which levels are shown in Table 4. Two of which, namely machine and material, are treated as categorical features, so using scikit-learn's OrdinalEncoder these features were encoded into an integer array. While the remaining three features were treated as continuous features, for standardizing these features scikitlearn's StandardScaler was used. It transformed the data so that for each feature, its distribution will have a mean value of 0 and a standard deviation of 1 . The formula used for this is as follows Eq (1). 
Table 4 Feature and their levels

\begin{tabular}{lcccccc}
\hline \multicolumn{1}{c}{ Feature } & Level 1 & Level 2 & Level 3 & Level 4 & Level 5 & Level 5 \\
\hline Blank Material & JSC 440 & JCS 590 & JSH 440 & JSH 590 & & \\
\hline Thickness (t) $\mathbf{m m}$ & 1.00 & 1.20 & 1.40 & 1.60 & \\
\hline Width (w) $\mathbf{m m}$ & 20 & 50 & & & \\
\hline Bend angle (A) \\
degree
\end{tabular}

Where $X$ is the sample, $Z$ is the standard score, $U$ is the mean of the training samples, and $\mathrm{S}$ is the standard deviation of the training samples.

After preprocessing data, the dataset is divided for training and testing using scikit-learn's train_test_split into a ratio of 9:1.

\subsection{Model Selection}

For this research paper, tree-based learning algorithms are used. These algorithms provide high precision, consistency, and interpretability to predictive models. They map non-linear relationships very well, unlike linear models. They can adapt to any situation and solve any problem. The data type is not a constraint to these models as these models can map both continuous and categorical data. The list of tree-based learning algorithms used in this paper are mentioned in Table 5.

Table 5 Models used for the machine learning

\begin{tabular}{lll}
\hline S.No. & \multicolumn{1}{c}{ Model used } & From libraries \\
\hline 1 & Decision tree & Scikit-learn \\
2 & Random forest & Scikit-learn \\
3 & Extra tree & Scikit-learn \\
4 & Light gradient boosting machine (lightgbm) & Lightgbm \\
5 & Gradient boosting & Scikit-learn \\
\hline
\end{tabular}

\subsection{Model training}

With five predictor variables and one target variable, five models are trained. For training decision tree, random forest, extra tree and Gradient boosting models, scikit learn was used, while for training Light gradient boosting machine (LightGBM) its library was used. Hyperparameter tuning of decision tree and Gradient boosting was done using hit and trial method while flaml was used for the random forest, extra tree and lightGBM. The best parameters are mentioned in Table 6. 
Table 6 Best parameters

\begin{tabular}{|c|c|}
\hline Model name & Parameters used \\
\hline Decision Tree & $\begin{array}{l}\text { max_depth=4, } \\
\text { min_samples_leaf }=8, \\
\text { min_weight_fraction_leaf }=0.0, \\
\text { max_leaf_nodes }=10000, \\
\text { ccp_alpha=0.0, } \\
\text { criterion=mae }\end{array}$ \\
\hline Random Forest & $\begin{array}{l}\text { bootstrap: True, } \\
\text { criterion: mse, } \\
\text { min_samples_leaf: } 1, \\
\text { min_samples_split: 2, } \\
\text { max_features=0.5119924258446656, } \\
\text { n_estimators }=35, \\
\text { n_jobs }=-1\end{array}$ \\
\hline LightGBM & $\begin{array}{l}\text { colsample_bytree=0.9502671771869313, } \\
\text { learning_rate=0.23684764381992687, } \\
\text { max_bin=127, } \\
\text { min_child_samples=21, } \\
\text { n_estimators=49, } \\
\text { num_leaves=19, } \\
\text { objective='regression', reg_alpha=0.24248019672976737, } \\
\text { reg_lambda=0.0009765625, subsample=0.6374486870608949 }\end{array}$ \\
\hline Extra Tree & $\begin{array}{l}\text { bootstrap: False, } \\
\text { max_features }=0.6170076481831065, \\
\text { n_estimators }=100, \\
\text { n_jobs=-1 } \\
\text { criterion: mse, } \\
\text { min_samples_leaf: } 1, \\
\text { min_samples_split: } 2\end{array}$ \\
\hline Gradient Boosting & $\begin{array}{l}\text { alpha: 0.1, } \\
\text { ccp_alpha: 0.0, } \\
\text { criterion: friedman_mse, } \\
\text { learning_rate: 0.1, } \\
\text { loss: ls, } \\
\text { max_depth': 3, } \\
\text { max_features: None, } \\
\text { max_leaf_nodes: None, } \\
\text { min_impurity_decrease: 0.0, } \\
\text { min_impurity_split: None, } \\
\text { min_samples_leaf: 1, } \\
\text { min_samples_split: 2, } \\
\text { min_weight_fraction_leaf: 0.0, } \\
\text { n_estimators: 100, } \\
\text { subsample: 1.0, } \\
\text { tol: 0.0001, } \\
\text { validation_fraction: } 0.1\end{array}$ \\
\hline
\end{tabular}

\subsection{Model Evaluation:}

For assessing the performance of the models, the available standard statistical measure of assessment has been used. Mean square error (MSE), mean absolute error (MAE), and root mean square error (RMSE) are determined to assess the accuracy of the models. Following are their definitions: 


$$
\begin{aligned}
& M A E=\frac{1}{N} \sum_{i=1}^{N}\left|e_{i}\right| \\
& M S E=\frac{1}{N} \sum_{i=1}^{N} e_{i}{ }^{2} \\
& R M S E=\sum_{i=1}^{N} \sqrt{M S E}
\end{aligned}
$$

Here $e_{i}$ (prediction error) represents the difference between actual and predicted value; $y_{i}$ represents the value observed from the data acquisition setup, and $N$ denotes the number of samples in the testing data set. Typically, the test set, also known as the unseen data to the model, are run, and these metrics are computed. Given the application requirements, each metric has benefits and drawbacks. For analyzing the performance of springback models, two metrics, namely $M A E$ and $M S E$ are used, in this research work.

\subsection{Model Working}

Since the foundation of all tree-based models is the decision tree, understanding this model will help us understand others. For training the decision tree, the data generated by the experimental setup have been used. In training, a decision tree makes decision splits iteratively and keeps branching out till a terminating condition is met. It is done by employing the binary recursive partitioning method as coded in python's scikit learn library, which is an optimized CART algorithm version. This branching (decision split) of a tree is at every node is made with respect to the predictor variable that produces the maximum information gain at each branch node, whereby the information gain is defined as the change in entropy before and after a decision split. As a result, the most crucial predictor variables are anticipated to emerge at the upper part of the tree, possibly many times; and insignificant predictor variables may appear sparingly at the lower tier or not at all. For example, if the Initial angle is a critical factor in predicting the springback, the tree will split so that this variable will be near the top. If this variable has an insignificant influence on springback, then it may appear in the lower tier of the tree or not appear at all in the trained model.

One problem with a decision tree is that it is unstable, as a slight change in the input dataset can significantly impact the final results, so in some instances, a single decision tree model would not be able to make accurate predictions on its own, to mitigate this issue several trees can be created and their predictions can be combined to boost the predictive capabilities of our model, this is known as ensembling. Ensembling refers to any set of models, but it is most commonly associated with tree-based models. Ensembling is like aggregating the results from many models to get better accuracy and stability. All tree-based algorithms other than decision trees are based on ensembling. There are two Ensembling methods known as bagging and boosting.

Bagging is a technique for creating various decision tree models, all at once by randomly sampling with replacement from the original dataset. It means that it can end up having variety in trees, which helps prevent overfitting. Random forest and extra tree are some of the famous bagging algorithms.

Boosting is an ensemble tree approach that constructs a series of small trees, often with only one node, with each tree focusing on correcting the previous tree's net error. As a result, the first tree will be disintegrated on the most predictive feature, then adjust weights to ensure that the subsequent tree splits on whatever feature helps it to correctly identify the data points that were misclassified in the first tree. The following tree will concentrate on correctly classifying errors from the previous tree, and so on. The final forecast is a weighted average of all individual forecasts. LightGBM and Gradient boosting are some of the famous extensions of boosting. 


\section{Discussion}

The dataset has been processed through the machine learning model, which trains the model and then performs validation. Following is the discussion over the evaluated results.

\subsection{Model performance}

Using the performance metrics defined in the methodology section, it is found that the best model for the prediction of springback is LightGBM which had a MAE and MSE of 0.41 and 0.25 , respectively. It is followed by Gradient boosting from Scikit learn, which had a MAE and MSE of 0.66 and 0.75, respectively, as shown in Table 7 . Hence the best class of algorithm in tree-based learning for this problem is Gradient boosting algorithms.

Table 7 Model error

\begin{tabular}{lcc}
\hline \multicolumn{1}{c}{ model name } & MAE & MSE \\
\hline Lightgbm (L) & 0.41667 & 0.25689 \\
Gradient boosting (G) & 0.66322 & 0.75954 \\
Random forest (R) & 0.70593 & 0.97595 \\
Extra tree (E) & 0.77252 & 1.01941 \\
decision tree (D) & 0.91667 & 1.69012 \\
\hline
\end{tabular}

To further evaluate the model for accuracy, few data points are taken from the experimental data, which are shown in Table 8. The combination of features (parameter) is input in the developed model to predict the springback and compare it with the experimental results. Random data taken from the experimental results are shown in Table 8 . Each dataset is given a set number so that it can be tracked.

Table 8 Sample Data taken from the experiments for the validation of the model

\begin{tabular}{ccccccc}
\hline $\begin{array}{c}\text { Set } \\
\text { No. }\end{array}$ & $\begin{array}{c}\text { Thickness } \\
(\mathbf{m m})\end{array}$ & $\begin{array}{c}\text { Width } \\
(\mathbf{m m})\end{array}$ & $\begin{array}{c}\text { Initial Angle } \\
(\text { Degree })\end{array}$ & Material & Machine & Springback \\
\hline 1 & 1.2 & 20 & 60 & JSC440 & Hydraulic - 75 - 0 - 180 & 2.500 \\
2 & 1.6 & 50 & 60 & JSH590 & Hydraulic - 75 - 10 -- 180 & 1.458 \\
3 & 1.4 & 50 & 120 & JSC440 & Mechanical - 60 - U & 0.333 \\
4 & 1.4 & 20 & 90 & JSC440 & Mechanical - 60 - E & 0.208 \\
5 & 1.2 & 20 & 120 & JSC440 & Mechanical - 60 - U & -3.333 \\
6 & 1 & 20 & 60 & JSC590 & Mechanical - 60 - U & -0.833 \\
7 & 1.2 & 50 & 120 & JSC440 & Hydraulic - 75 - 10 - 30 & -3.083 \\
8 & 1 & 50 & 60 & JSC590 & Mechanical - 60 - E & 1.917 \\
9 & 1.2 & 20 & 60 & JSC440 & Hydraulic - 75 - 10 -- 180 & 2.250 \\
\hline
\end{tabular}

Using the developed models, the springback is predicted for the nine data points shown in Table 8 . The predicted springback for each data point is shown in Table 9 and labelled with the respective data points. It can be seen from the Table 9 that the MAE, MSE and RSME for the model ' $\mathrm{L}$ ' LightGBM is least as determined for the whole dataset. Hence the LightGBM model is most suitable for the prediction of springback. 
Table 9 Sample predictions from Models vs Actual error

\begin{tabular}{ccccccccccc}
\hline Set & R & Dev. & G & Dev. & L & Dev. & E & Dev. & D & Dev. \\
\hline 1 & -0.280 & 2.78 & 0.682 & 1.818 & 1.650 & 0.85 & 1.876 & 0.624 & -1.417 & 3.917 \\
2 & 0.972 & 0.486 & 0.440 & 1.018 & 1.148 & 0.31 & 0.020 & 1.438 & 0.396 & 1.062 \\
3 & 0.310 & 0.023 & 0.596 & -0.263 & 0.614 & -0.281 & 1.393 & -1.06 & -1.000 & 1.333 \\
4 & 1.418 & -1.21 & 0.680 & -0.472 & 1.051 & -0.843 & 1.409 & -1.201 & 1.750 & -1.542 \\
5 & -3.977 & 0.644 & -3.768 & 0.435 & -3.132 & -0.201 & -4.344 & 1.011 & -3.417 & 0.084 \\
6 & -0.453 & -0.38 & 0.129 & -0.962 & -0.662 & -0.171 & -1.027 & 0.194 & 0.396 & -1.229 \\
7 & -2.767 & -0.316 & -3.620 & 0.537 & -2.895 & -0.188 & -2.729 & -0.354 & -3.417 & 0.334 \\
8 & 2.732 & -0.815 & 1.984 & -0.067 & 1.661 & 0.256 & 2.127 & -0.21 & 0.396 & 1.521 \\
9 & -0.765 & 3.015 & -0.210 & 2.46 & 1.215 & 1.035 & -0.051 & 2.301 & -1.417 & 3.667 \\
& MAE & 1.074 & MAE & 0.892 & MAE & 0.459 & MAE & 0.932 & MAE & 1.632 \\
& MSE & 2.206 & MSE & 1.344 & MSE & 0.317 & MSE & 1.283 & MSE & 4.223 \\
& RSME & 1.485 & RSME & 1.159 & RSME & 0.563 & RSME & 1.133 & RSME & 2.055 \\
\hline
\end{tabular}

Fig 5 show the predicted values of springback vs actual springback for all models; this figure also indicated that LightGBM gave best prediction to test data (unseen by model). 


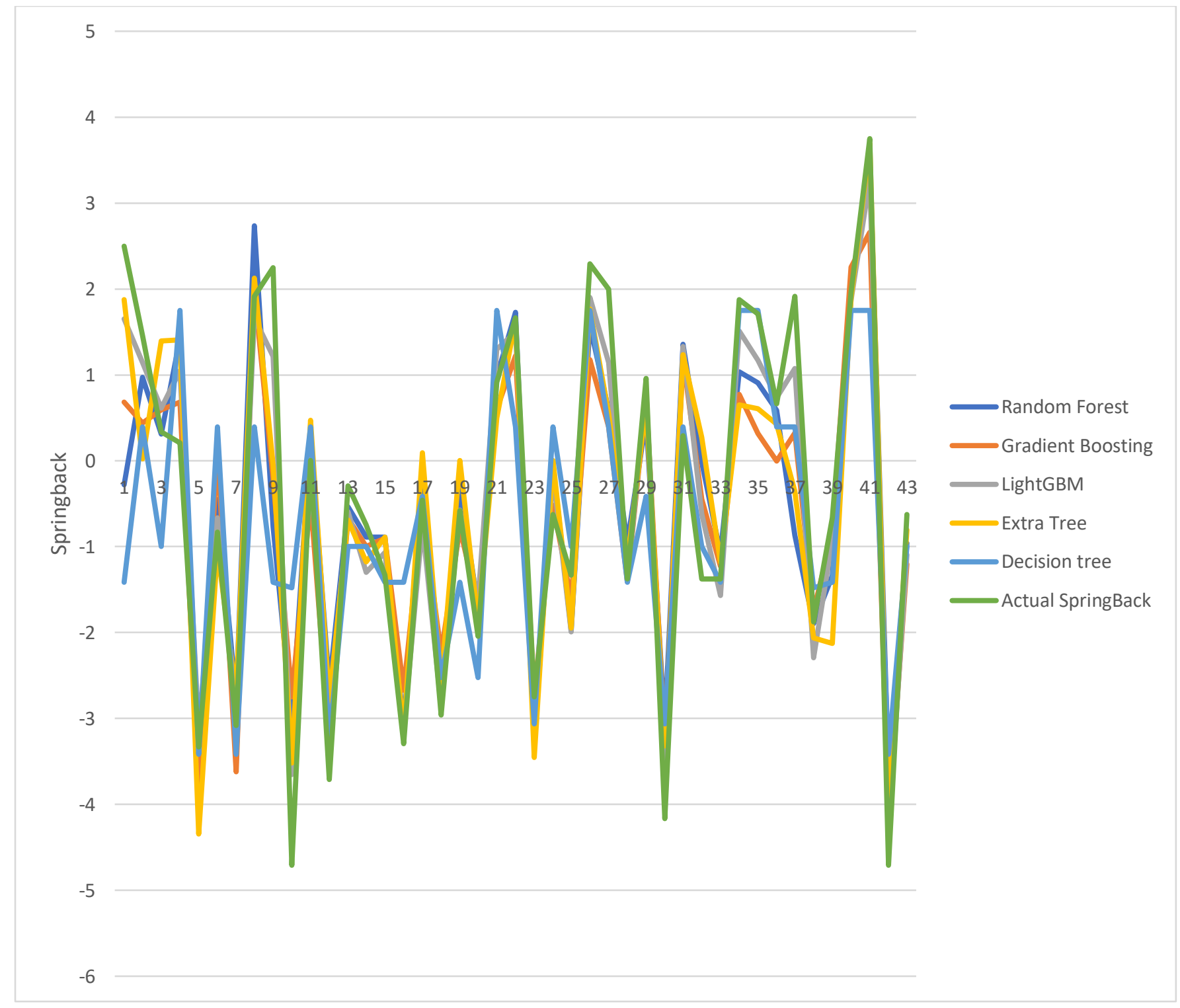

Fig 5 Actual springback vs springback predicted by models for test data

\section{Conclusion}

This paper introduced tree-based learning for the prediction of springback, which provides high precision, consistency, and interpretability to predictive models. The data for training these models on was collected by conducting experimental studies using the experimental setup. After collecting the data, the data is prepared by preprocessing it. It is then randomly divided into a ratio of $9: 1 ; 90 \%$ of the data was used for training while rest for testing.

After evaluating the performance of Random Forest, Gradient Boosting, LightGBM, Extra Tree and Decision tree algorithms it was determined that the most acceptable results were delivered by lightGBM with a certain combination of parameters that were acquired by fine-tuning the model using Flaml, and it was found that on test data (unseen data) it had a MAE and MSE of 0.41 and 0.25 respectively. These results demonstrated a good agreement between predicted and measured values. Therefore, LightGBM can be used for the prediction of springback in a manufacturing setup. 
This model can fulfill the immense need of the industry to predict springback in high-tensile strength steel for a combination of parameters (features) off-line. Furthermore, more data sets can be entered into the model to train it for other types of sheet metals as future work. More features can also be incorporated to add more certainty to the model.

\begin{abstract}
Authors contributions:
This article is a result from collaboration between scientists. The corresponding author, Saad Ur Rehman Baig, has been responsible for the conceptualizing, planning, and developing the idea, along with implementing the code, conducting this research and writing the publication. Muhammad Wasif has been responsible for conceptualizing, data collection, editing, and as well as in structuring this article. Anis Fatima has been responsible for reviewing and corrections. Mirza Muhammad Anas Baig supported in reviewing and validation. Syed Amir Iqbal supported in research coordination and supervision.

Funding: funding has been provided by the NED university of engineering and technology.

Availability of data and other material: The data and other material will be made available upon reasonable request and cost.

Declarations

Conflicts of interest: The authors declare no conflicts of interest.

Ethics approval and consent to participate: Not applicable.

Consent to publish: All authors declare that they agree with the publication and consent to publish this paper.
\end{abstract}

\title{
References
}

[1] M. P. Groover, Fundamentals of Modern Manufacturing: Materials, Processes, and Systems, 6th Edition: Edition 6, Hoboken, New Jersey: Wiley, 2016.

[2] M. Wasif, S. A. Iqbal, M. Tufail and H. Karim, "Experimental Analysis and Prediction of Springback in V-bending Process of High-Tensile Strength Steels," Trans Indian Inst Met 73, pp. 285-300, 2020.

[3] S. Kalpakjian and S. Schmid, Manufacturing Engineering and Technology (7th), London: Pearson Higher Ed., 2013.

[4] W. D. Cardena, L. M. Genga, D. K. Matlockb and R. H. Wagoner, "Measurement of springback," International Journal of Mechanical Sciences, vol. 44, p. 79, 2002.

[5] Z. Tekinar, "An experimental study on the examination of springback of sheet metals with several thicknesses and properties in bending dies.," J Mater Process Tech, vol. 145, pp. 109117, 2004.

[6] D. Fei and P. Hodgson, "Experimental and numerical studies of springback in air v-bending process for cold rolled TRIP steels.," Nucl Eng Des, vol. 236, no. 18, pp. 1847-1851, 2006.

[7] Z. Dong-juan, C. Zhen-shan, C. Zhi-ying and R. Xue-yu, "An analytical model for predicting sheet springback after V-bending," J Zhe- jiang Univ-SC A, vol. 8, no. 2, pp. 237-244, 2007.

[8] F. Ozturk, S. Toros and S. Kilic, "Tensile and Spring-Back Behavior of DP600 Advanced High Strength Steel at Warm Temperatures.," J. Iron. Steel. Res. Int., vol. 16, no. 6, pp. 41-46, 2009.

[9] M. V. Inamdar, P. P. Date and S. V. Sabnis, J Mater Process Tech, vol. 123, p. 459, 2002.

[10] R. Z. M. a. A. R. Ramezani M, "Modelling of kinetic friction in V-bending of ultra-highstrength steel sheets.," Int. J. Adv. Manuf. Tech., vol. 46, no. 3, pp. 101-110, 2010.

[11] a. B. P. Swiotoniowski A, J KONES Powertrain Transp, vol. 19, p. 459, 2012.

[12] J. Slota and M. Jurcisin, "Experimental and numerical prediction of springback in v-bending of anisotropic sheet metals for automotive industry," Zeszyty Naukowe Politechniki Rzeszowskiej. Mechanika, vol. 284, pp. 55-67, 2012. 
[13] E. A. Da Silva, L. F. Fernandes, J. W. Silva, R. B. Ribeiro, M. D. Pereira and J. Alexis, "A Comparison between an Advanced High-Strength Steel and a High-Strength Steel Due to the Springback Effect.," IOSR J. Mech. Civ. Eng., vol. 13, no. 5, pp. 21-27, 2016.

[14] Y. X, C. Choi, N. K. Sever and T. Altan, "Prediction of springback in air-bending of Advanced High Strength steel (DP780) considering Young's modulus variation and with a piecewise hardening function," Int. J. Mech. Sci., vol. 10, pp. 266-272, 2016.

[15] J. Jung, S. Jun, H. S. Lee, B. M. Kim, M. G. Lee and J. H. Kim, "Anisotropic Hardening Behaviour and Springback of Advanced High-Strength Steels," Metals, vol. 7, no. 11, pp. 1-14, 2017.

[16] J. Choi, J. Lee, H. J. Bong, M. G. Lee and F. Barlat, "Advanced constitutive modeling of advanced high strength steel sheets for springback prediction after double stage U-draw bending," Int. J. Solids. Struct., vol. 151, pp. 152-164, 2018.

[17] D. K. Leu and Z. W. Zhuang, "Springback prediction of the vee bending process for highstrength steel sheets," J Mech Sci Technol, vol. 30, no. 3, pp. 1077-1084, 2016.

[18] Z. H. Cai, P. Batthya' ny, S. Dhawan, Q. L. Zhang, Y. H. Sun, X. Luan, L. L. Wang and M. M. Gharbi, "Study of Springback for High Strength Aluminium Alloys Under Hot Stamping, Advanced High Strength Steel and Press Hardening.," Adv. High Strength Steel Press Hardening, vol. 1, pp. 117-121, 2019.

[19] Y. Nakagawa, K. Mori and T. Maeno, "Springback-free mechanism in hot stamping of ultrahigh-strength steel parts and deformation behaviour and quenchability for thin sheet.," Int. J. Adv. Manuf. Tech., vol. 95, no. 1-4, pp. 456-467, 2018.

[20] N. Noma, K. Hashimoto, T. Maeda and T. Kuwabara, "High accuracy springback simulation by using material model considering the SD effect.," J. Phys. Conf. Ser., vol. 1063, pp. 1-4, 2018.

[21] C. G. Liu, T. Yue and X. T. Yu, "Springback Analysis of the Stiffened Panel Milling from the Bent Plate.," Int. J . Precis. Eng. Man., vol. 20, no. 2, pp. 217-232, 2019.

[22] C. Liu, X. D. Yan, Y. Yang and W. Ye, "Research on the Springback of Square TA18 Titanium Alloy Tube on the Numerical Simulation," Mater. Sci. Forum., vol. 944, pp. 753-760, 2019.

[23] R. Ramadass, S. Sambasivam and K. Thangavelu, "Selection of optimal parameters in Vbending of Ti-Grade 2 sheet to minimize springback.," J. Braz. Soc. Mech. Sci., vol. 41, p. 21, 2018.

[24] J. Lin, Y. Hou, J. Min, H. Tang, J. E. Carsley and T. B. Stoughton, "Stoughton TB 2019 Effect of constitutive model on springback prediction of MP980 and AA6022-T4," Int. J. Mater. Form., vol. 13, pp. 1-13, 2019.

[25] M. Inamdar, P. Date, K. Narasimhan, S. K. Maiti and U. P. Singh, "Development of an Artificial Neural Network to Predict Springback in Air Vee Bending," Int J Adv Manuf Technol, no. 16, p. 376-381, 2000.

[26] M. Dezelak, I. Pahole, M. Ficko and M. Brezocnik, "MACHINE LEARNING FOR THE IMPROVEMENT OF SPRINGBACK MODELLING," Advances in Production Engineering \& Management, no. 7(1), pp. 17-26, 2012.

[27] R. Narayanasamy and P. Padmanabhan, "Comparison of regression and artificial neural network model for the prediction of springback during air bending process of interstitial free steel sheet," J Intell Manuf, p. 357-364, 2012.

[28] R. Kazan, M. Firat and A. Tiryaki, "Prediction of springback in wipe-bending process of sheet metal using neural network," Materials \& design, pp. 418-423, 2009.

[29] M. Bozdemir and M. Golcu, "Artificial neural network analysis of springback in V bending," $J$ Appl Sci, vol. 17, no. 8, pp. 3038-3043, 2008. 